\title{
Referentenentwurf zum PsychVVG: Wir sind auf einem guten Weg - Kontra
}

\author{
Ministerial Draft Bill for the Law on Remuneration of Inpatient Psychiatry and \\ Psychosomatics (Psych VVG): We are Headed in the Right Direction - Contra
}

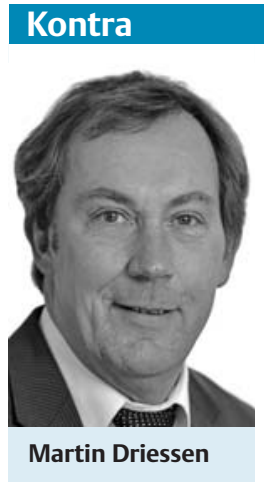

Die zentrale Frage, die uns in der Debatte um den aktuellen Referentenentwurf beschäftigen muss, ist doch: Hilft ein solches Gesetz (wenn es denn so Gesetz würde), die psychiatrisch-psychosomatisch-psychotherapeutische Versorgung der Betroffenen, insbesondere der schwer Kranken, in eine sinnvolle Richtung weiter zu entwickeln? Die Beantwortung hängt natürlich entscheidend von den definierten Zielen ab, über die sich ebenfalls trefflich diskutieren lässt. Aus Sicht (nicht nur) des Autors seien hier einige genannt und gefragt, ob der Entwurf hilfreiche Antworten und Lösungen verspricht.

1. Werden qualitative Verbesserungen der (teil-)stationären Krankenhausversorgung erreicht? Wie überall in der Medizin hängt auch in unserem Fachgebiet der Behandlungserfolg vom Kennen und Können ab, d.h. wie gut sind unsere Mitarbeitenden ausgebildet? Aber dies nützt nur, wenn auch genügend hoffentlich sehr gut ausgebildete Mitarbeitende zur Verfügung stehen. Vor dem Hintergrund der enormen fachlichen Entwicklung in unserem Fachgebiet werden die Standards durch gültige Leitlinien, hohe ethisch-rechtliche Ansprüche (Selbstbestimmungsrecht), bald Qualitätsindikatoren und berechtigte Ansprüche unserer Patienten und ihrer Angehörigen gesetzt. Ich kenne keine FachkollegInnen, egal welcher Profession, die meinen, dass man diesen Standards unterhalb einer PsychPV-Erfüllung von 110\% auch nur annähernd gerecht werden kann (für diese Erkenntnis muss man nicht von organisierter Kunstfehlerpsychiatrie sprechen) - oder einer entsprechenden Personalausstattung auf der Grundlage eines weiterentwickelten Personalbemessungsinstruments. Dass eine solche Marge angestrebt wird, davon ist in diesem Entwurf nicht die Rede, wenn man die Details liest.

2. Flexibilisiert der Entwurf die Krankenhauspsychiatrie in Richtung einer den persönlichen Behandlungsbedarfen angepassten und vom Bett unabhängigen Behandlungseinrichtung? Wir sollten uns erinnern: Kein Patient geht gerne ins Krankenhaus und unter fachlichen Gesichtspunkten ist eine Behandlung mit Bett heute häufig auch nicht notwendig, wenn es denn finanzierte Alternativen gäbe. Andererseits ist eine hochintensive Krankenbehandlung außerhalb des Krankenhauses aufwendiger als innerhalb. Daher muss es das Ziel sein, eine Krankenhausbehandlung zu ermöglichen, die flexibel und mit abgestufter Intensität Angebote bereitstellt, ohne das System (auch finanziell) zu überfordern. Der derzeitige Referentenentwurf erfüllt nahezu keine dieser Bedingungen. Er schreibt dagegen ein starres System von (stationsäquivalentem?) Hometreatment vor, das jederzeit alle Elemente einer vollstationären Behandlung (mit deutlich höherem personellem Aufwand) realisieren soll - und damit praktisch unmöglich wird.

3. Ermöglicht der Entwurf eine intensiv ambulante Behandlung durch das Krankenhaus? Jenseits einer stationsersetzenden flexiblen Krankenhausbehandlung fehlt in Deutschland die (regelfinanzierte) Möglichkeit einer intensiv-ambulanten Behandlung unterhalb der tagesklinischen Schwelle - mit einer relativen Ausnahme, dem bayerischen Modell der Dokumentation und Vergütung der psychiatrischen Institutsambulanzen (relativ, weil es auch hier auf die Vergütung ankommt). Für viele Patienten ist in akuten und subakuten Krankheitszeiten eine solche Behandlungsmodalität sehr wertvoll, weil sie (teil-)stationäre Krankenhausbehandlung verhindern kann und zu- dem auch kostengünstiger ist. Zwar finden sich in der Begründung zum Referentenentwurf Hinweise darauf, dass die Bedeutung der Psychiatrischen Institutsambulanzen für die Versorgung künftig steigen wird, dabei wird allerdings lediglich auf den SGB V §115d Absatz 1 (neu) abgehoben, wonach die stationsäquivalenten Leistungen auch an die PIA delegiert werden können.

4. Unterstützt der Entwurf die in unserem Fachgebiet notwendige Netzwerkarbeit? Krankenhauspsychiatrie insbesondere für schwer und chronisch Kranke hat immer ein davor und danach und findet daher in einem gemeindepsychiatrischen Kontext statt, die einen systematischen, ständigen und intensiven Austausch mit allen relevanten Einrichtungen der allgemeinpsychiatrischen, psychotherapeutischen, Sucht- und Altenhilfe inklusive Selbsthilfe und Angehörigenverbänden erfordert. Der (im Übrigen nach wie vor weitgehend unbestimmte) Begriff der Pflichtversorgung findet sich zwar im Referentenentwurf als budgeterhöhendes Merkmal, beschreibt die mit dieser Netzwerkarbeit verbundenen Tätigkeiten und Aufwände und Personalbindung aber unvollständig.

5. Ist der Entwurf in sich schlüssig? Im Vorabtext wird vielversprechend formuliert, dass Gesetzentwurf auf die Weiterentwicklung der Versorgung und der Vergütung für psychiatrische und psychosomatische Leistungen abzielt. Ziel sei es, die sektorenübergreifende Behandlung in der psychiatrischen Versorgung zu fördern sowie die Transparenz und die Leistungsorientierung der Vergütung zu verbessern. Tatsächlich geht es aber im Gesetzestext überwiegend um sicher wichtige Vergütungsfragen, die sich aber wiederum überwiegend um konkrete Fragen des PEPP-Systems drehen, während weitere budgetrelevante Fragen sehr im ungefähren bleiben bzw. an andere Instanzen delegiert werden (s.u.). Unbe- 
stimmt bleibt auch das Verhältnis von Krankenhausvergleichen auf Landesund Bundesebene und die Budgetverhandlung vor Ort. $\mathrm{Zu}$ befürchten ist, dass es eben doch zu einer Mittelwertanpassung (im schlimmsten Fall auf Mangelniveau) kommen wird. Vor allem aber fehlt im Gesetzestext die klare inhaltliche Zielvorstellung zur Verbesserung der Versorgung, die mit den einzelnen Vorgaben verknüpft ist. Dies führt unmittelbar zur nächsten Frage.

6. Schafft der Entwurf Rechtssicherheit? Trotz der positiven Absichten (Budgetsystem, verbindliche Personalausstattung, Home treatment) verweist der Entwurf an zahlreichen Stellen auf noch zwischen den Selbstverwaltungspartnern (zwischen DKG und GKV Spitzenverband oder zusätzlich der KBV) zu verhandelnde Details (z.B. welche Leistungen denn nun das Hometreatment genau definieren). Wer solche Verhandlungen kennt, weiß, dass dies ein zäher und langwieriger Verhandlungsmarathon werden wird, der mit zahlreichen Möglichkeiten der Verzö- gerung verbunden ist. In dieser Zeit (Jahre?) wird es zu zahlreichen Punkten sicher keine Rechtssicherheit geben. Auch was die Budgetregelungen angeht, ist für die Verhandlungen vor Ort die Tür für zahlreiche Schiedsstellen- und Gerichtsverfahren weit geöffnet. Letztlich führt dies - und das ist das wesentliche Problem für die Kliniken vor Ort - zu erheblichen Verzögerungen der Weiterentwicklung der Versorgung.

Zusammenfassend und etwas plakativ formuliert: Die Verpackung stimmt, der Inhalt an etlichen Stellen nicht. Trotz unbestreitbarer positiver Absichten und Ansätze des Referentenentwurfs zu einer dringend notwendigen Weiterentwicklung der Psychiatrie und Psychosomatik in Deutschland sind die vorgeschlagenen Inhalte im Detail für diese Absichten nicht förderlich oder werden womöglich das Gegenteil bewirken. Man gewinnt etwas den Eindruck, dass die historische Chance für eine echte Verbesserung der Behandlung und Versorgung vertan zu werden droht. $\mathrm{Zu}$ wünschen ist, dass die verant- wortlichen Politiker den Mut aufbringen, klare Akzente im Sinne der psychisch Kranken zu setzen, auch wenn dies mit einem höheren finanziellen Aufwand verbunden ist.

Sie haben eine eigene Meinung zu diesem Thema? Dann schreiben Sie uns an: psychiat-praxis@thieme.de!

\section{Korrespondenzadresse}

\section{Prof. Dr. Martin Driessen}

Chefarzt und Ärztlicher Direktor, Klinik für Psychiatrie und Psychotherapie, Ev. Krankenhaus Bielefeld

Remterweg 60-71

33649 Bielefeld

martin.driessen@evkb.de

\section{Bibliografie}

DOI http://dx.doi.org/

10.1055/s-0042-112247

Psychiat Prax 2016; 43: 303-304

(c) Georg Thieme Verlag KG

Stuttgart · New York

ISSN 0303-4259 